\title{
An Isolable Magnesium(II)-Radical Dianion Complex
}

\author{
Jinjing Pan, ${ }^{\dagger}$ Li Zhang, ${ }^{\prime \ddagger}$ Yuhao He, ${ }^{\dagger}$ Qian Yu, ${ }^{\dagger}$ Gengwen Tan ${ }^{\dagger *}$ \\ ${ }^{\dagger}$ College of Chemistry, Chemical Engineering and Materials Science, Soochow University, Suzhou \\ 215123, China. \\ "Center of Materials Science and Engineering, Guangxi University of Science and Technology, \\ Liuzhou 545006, China. \\ E-mail: gwtan@suda.edu.cn; ${ }^{\ddagger}$ J.P. and L.Z. contributed equally to the work.
}

\section{Content}

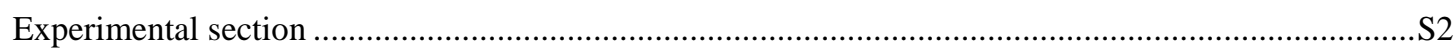

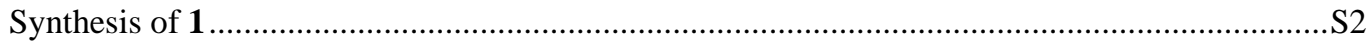

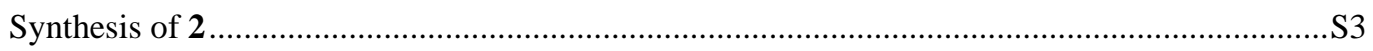

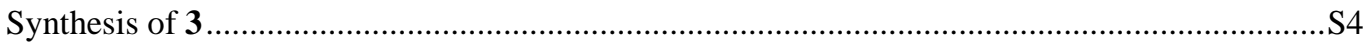

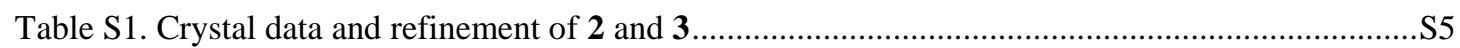

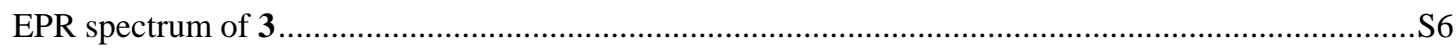

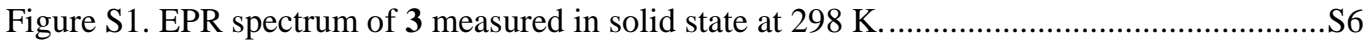

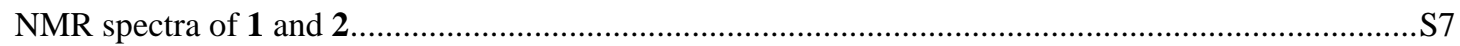

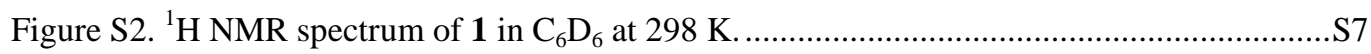

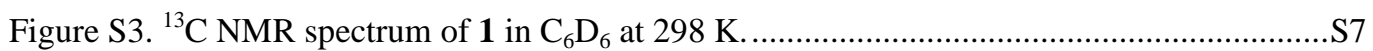

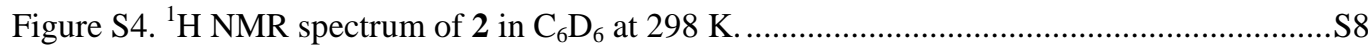

Table S2 Calculated absorption properties of the studied compound including wavelength (nm),

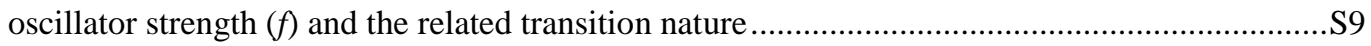

Table S3 Calculated Kohn-Sham orbitals related to the transitions ........................................S10 


\section{Experimental section}

General considerations: All experiments were carried out under dry oxygen-free nitrogen using standard Schlenk techniques or in a $\mathrm{N}_{2}$ filled-glove box. Solvents were dried by standard methods and stored in activated $4 \AA$ molecule sieve in the glovebox. The NMR spectra were recorded on Bruker spectrometers (AV400) referenced to residual solvent signals as internal standards. The solutions of samples in the deuterated solvent were sealed off in a NMR tube under vacuum for measurements. UV/Vis spectra were recorded on the Lambda 750 spectrometer at room temperature. Element analyses were performed on an ElementarVario EL III instrument. EPR spectra were obtained using JEOL JES-X320 X-band variable-temperature apparatus. For the single crystal X-ray structure analyses the crystals were each mounted on a glass capillary in perfluorinated oil and measured in a cold $\mathrm{N}_{2}$ flow. The data for all compounds were collected on a Bruker D8 CMOS detector at low temperatures. The structures were solved by direct methods and all refined on $F^{2}$ with the SHELX-2018/3 software package. The positions of the $\mathrm{H}$ atoms were calculated and considered isotropically according to a riding model. SQUEEZE was used to remove the highly disordered solvent molecules in the crystal structure of $\mathbf{3}$ (2.5 THF in one asymmetric unit). Commercially available reagents were purchased from Energy Chemical and used as received.

\section{Synthesis of 1}
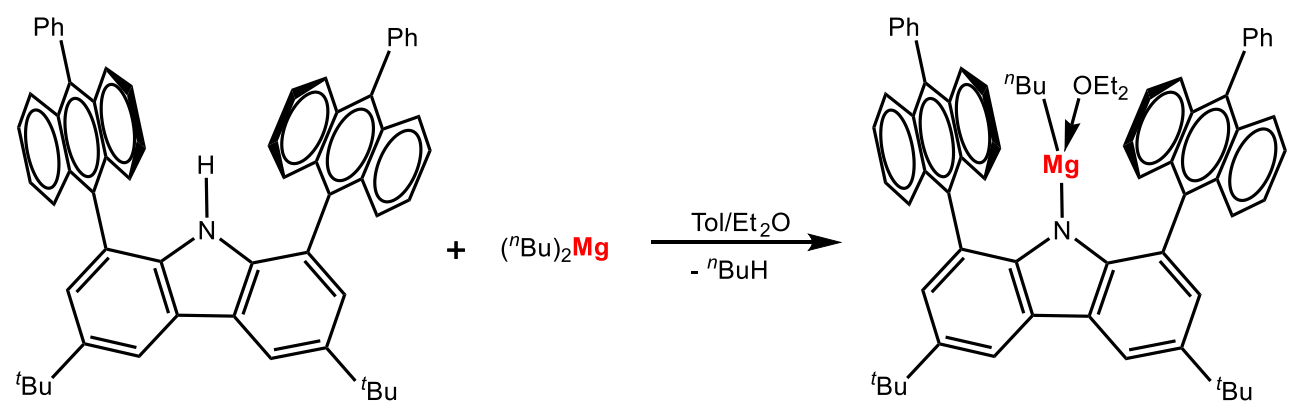

Dibutyl magnesium (4.00 mL, $1 \mathrm{M}$ in heptane/Et ${ }_{2} \mathrm{O}, 4.00 \mathrm{mmol}, 2$ eq.) was added dropwise to a solution of $\mathrm{LH}\left(1.57 \mathrm{~g}, 2.00 \mathrm{mmol}, 1\right.$ eq.) in anhydrous toluene $(20 \mathrm{~mL})$ at room temperature under $\mathrm{N}_{2}$ atmosphere. The mixture was stirred for $2 \mathrm{~d}$. The formed precipitate was isolated byfiltration, and was washed with $n$-hexane $(3 \times 5 \mathrm{~mL})$. The obtained yellow powder of 1 was dried under vacuum for $1 \mathrm{~h}$. Yield: $1.33 \mathrm{~g}, 1.41 \mathrm{mmol}, 71 \%$. Crystals suitable for single-crystal X-ray diffraction analysis were grown from toluene/n-hexane solution at room temperature. ${ }^{1} \mathrm{H}$ NMR $\left(\mathrm{C}_{6} \mathrm{D}_{6}, 400 \mathrm{MHz}, 298 \mathrm{~K}, \mathrm{ppm}\right): \delta$ $=-2.56-2.52\left(\mathrm{~m}, 2 \mathrm{H}, \mathrm{Mg}-\underline{\mathrm{H}}_{2}\right),-0.48-0.40\left(\mathrm{~m}, 2 \mathrm{H}, \mathrm{Mg}-\mathrm{CH}_{2} \underline{\mathrm{C}}_{2}\right),-0.10\left(\mathrm{t},{ }^{3} J_{\mathrm{H}, \mathrm{H}}=6.4 \mathrm{~Hz}, 6 \mathrm{H}\right.$, 
$\left.\mathrm{MgO}\left(\mathrm{CH}_{2} \underline{\mathrm{C}}_{3}\right)_{2}\right), 0.67-0.70\left(\mathrm{~m}, 3 \mathrm{H}, \mathrm{Mg}-\left(\mathrm{CH}_{2}\right)_{3} \mathrm{C}_{3}\right), 0.76-0.89\left(\mathrm{~m}, 2 \mathrm{H}, \mathrm{Mg}-\mathrm{CH}_{2} \mathrm{CH}_{2} \mathrm{C}_{2} \mathrm{CH}_{3}\right), 1.57$ (s, 18H, C $\left.\left(\underline{\mathrm{C}}_{3}\right)_{3}\right), 2.18\left(\mathrm{q},{ }^{3} J_{\mathrm{H}, \mathrm{H}}=6.4 \mathrm{~Hz}, 4 \mathrm{H}, \mathrm{MgO}\left(\mathrm{C}_{2} \mathrm{CH}_{3}\right)_{2}\right), 6.96-6.99$ (m, 9H, Ar- $\left.\underline{H}\right), 7.23-7.33(\mathrm{~m}$, $6 \mathrm{H}, \operatorname{Ar}-\underline{H}), 7.38-7.42(\mathrm{~m}, 2 \mathrm{H}, \operatorname{Ar}-\underline{H}), 7.62\left(\mathrm{~d},{ }^{4} J_{\mathrm{H}, \mathrm{H}}=1.7 \mathrm{~Hz}, 2 \mathrm{H}\right.$, carbazole- $\left.\underline{H}\right), 7.74-7.80(\mathrm{~m}, 6 \mathrm{H}$, $\operatorname{Ar}-\underline{H}), 8.07(\mathrm{br}, 3 \mathrm{H}, \mathrm{Ar}-\underline{H}), 8.90\left(\mathrm{~d},{ }^{4} J_{\mathrm{H}, \mathrm{H}}=1.7 \mathrm{~Hz}, 2 \mathrm{H}\right.$, carbazole- $\left.\underline{H}\right) ;{ }^{13} \mathrm{C}\left\{{ }^{1} \mathrm{H},{ }^{13} \mathrm{C}\right\} \mathrm{NMR}\left(\mathrm{C}_{6} \mathrm{D}_{6}, 100\right.$ $\mathrm{MHz}, 298 \mathrm{~K}$, ppm): $\delta=2.6\left(\mathrm{Mg}_{\underline{C}} \underline{\mathrm{H}} \mathrm{H}_{2}\right), 13.0\left(\mathrm{MgO}\left(\mathrm{CH}_{2} \underline{C} \mathrm{H}_{3}\right)_{2}\right), 14.3\left(\mathrm{Mg}_{-} \mathrm{CH}_{2} \underline{C} \mathrm{H}_{2}\right), 29.9$ $\left(\mathrm{Mg}-\mathrm{CH}_{2} \mathrm{CH}_{2} \mathrm{CH}_{2} \underline{\mathrm{C}} \mathrm{H}_{3}\right), \quad 32.1 \quad\left(\mathrm{Mg}-\mathrm{CH}_{2} \underline{C H}_{2} \mathrm{CH}_{2} \mathrm{CH}_{3}\right), \quad 32.6 \quad\left(\mathrm{C}\left(\underline{C} \mathrm{H}_{3}\right)_{3}\right), \quad 35.0 \quad\left(\underline{\mathrm{C}}\left(\mathrm{CH}_{3}\right)_{3}\right), \quad 64.4$ $\left(\mathrm{MgO}\left(\mathrm{CH}_{2} \mathrm{CH}_{3}\right)_{2}\right), 116.4,122.8,125.7,126.1,126.5,127.0,127.9,128.6,128.8,131.2,131.3,131.5$, 131.7, 134.9, 138.9, 139.5, 140.1, 149.5 (Ar- $\underline{-}$ ). Elemental analysis for $\mathrm{C}_{68} \mathrm{H}_{67} \mathrm{MgNO}(\%)$ : Calculated: C 87.02, H 7.20, N 1.49; Found: C 84.45; H 7.37; N 1.41. (Consistently low C analysis may be due to the formation of magnesium carbide).

\section{Synthesis of 2}
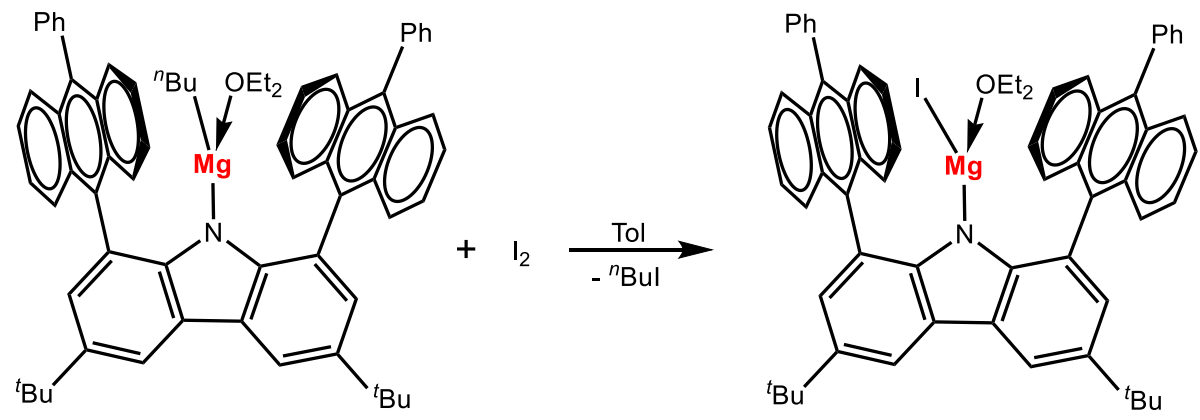

In a $\mathrm{N}_{2}$-filled glovebox, $\mathrm{I}_{2}(0.67 \mathrm{~g}, 2.63 \mathrm{mmol}, 1.05$ eq.) was added to a suspension of 1 ( $2.35 \mathrm{~g}, 2.50$ mmol, 1 eq.) in $30 \mathrm{~mL}$ of anhydrous toluene at $-30{ }^{\circ} \mathrm{C}$. After stirring for $2 \mathrm{~d}$ at room temperature, the mixture was filtered and the obtained precipitate was washed with $n$-hexane $(3 \times 5 \mathrm{~mL})$ to afford the yellow powder of 2, which was dried under vacuum for $1 \mathrm{~h}$. Yield: $2.20 \mathrm{~g}, 1.86 \mathrm{mmol}$, 74\%. Crystals suitable for single-crystal X-ray diffraction analysis were grown from toluene/ $n$-hexane solution at room temperature. ${ }^{1} \mathrm{H} \mathrm{NMR}\left(\mathrm{C}_{6} \mathrm{D}_{6}, 400 \mathrm{MHz}, 298 \mathrm{~K}, \mathrm{ppm}\right): \delta=-0.10\left(\mathrm{br}, 6 \mathrm{H}, \mathrm{MgO}\left(\mathrm{CH}_{2} \mathrm{C}_{3}\right)_{2}\right), 1.57$ (s, 18H, C(C $\left.\left.\underline{\mathrm{H}}_{3}\right)_{3}\right), 2.22$ (br, 4H, $\left.\mathrm{MgO}\left(\underline{C}_{2} \mathrm{CH}_{3}\right)_{2}\right), 6.94-7.00$ (m, 8H, Ar- $\left.\underline{H}\right), 7.21-7.27$ (m, 4H, Ar- $\left.\underline{H}\right)$, 7.32-7.37 (m, 5H, Ar- $\underline{H}), 7.64\left(\mathrm{~d},{ }^{4} J_{\mathrm{H}, \mathrm{H}}=1.8 \mathrm{~Hz}, 2 \mathrm{H}\right.$, carbazole- $\left.\underline{H}\right), 7.77-7.83(\mathrm{~m}, 5 \mathrm{H}, \mathrm{Ar}-\underline{H})$ 8.19-8.26 (m, $4 \mathrm{H}, \operatorname{Ar}-\underline{H}), 8.88\left(\mathrm{~d},{ }^{4} J_{\mathrm{H}, \mathrm{H}}=1.8 \mathrm{~Hz}, 2 \mathrm{H}\right.$, carbazole- $\left.\underline{H}\right)$. The ${ }^{13} \mathrm{C}$ NMR data was not obtained due to the low solubility in organic solvents, such as benzene and toluene. When THF- $\mathrm{d}_{8}$ was used, the coordinated diethyl ether was substituted by $T H F-d_{8}$ molecule. Elemental analysis for $\mathrm{C}_{71} \mathrm{H}_{66} \mathrm{IMgNO}$ (2•toluene) (\%): Calculated: C 77.49, H 6.05, N 1.27; Found: C 74.33; H 6.06; N 1.36. (Consistently low $\mathrm{C}$ analysis may be due to the formation of magnesium carbide). 


\section{Synthesis of 3}

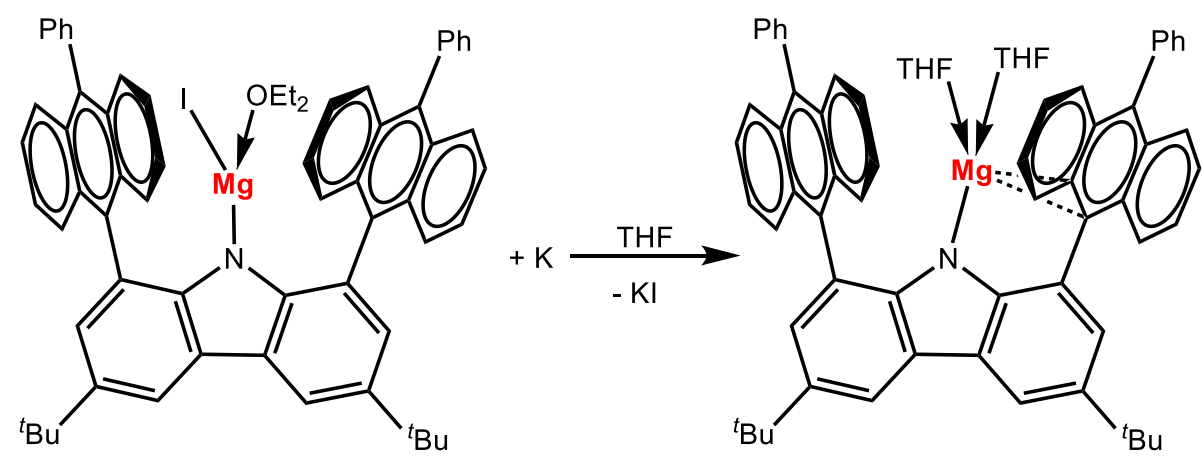

$2(2.37 \mathrm{~g}, 2.00 \mathrm{mmol}, 1$ eq.) and elemental potassium $(0.074 \mathrm{~g}, 1.90 \mathrm{mmol}, 0.95$ eq.) were placed in a $50 \mathrm{~mL}$ Schlenk flask, and THF $(20 \mathrm{~mL})$ was added at $-30{ }^{\circ} \mathrm{C}$. After stirring for $12 \mathrm{~h}$ at room temperature, the obtained deep green mixture was filtered. The filtrate was concentrated to ca. $10 \mathrm{~mL}$ and stored at $-30{ }^{\circ} \mathrm{C}$ for $12 \mathrm{~h}$ to afford deep green crystals of 3. Yield: $1.19 \mathrm{~g}, 1.26 \mathrm{mml}, 63 \%$. Elemental analysis for $\mathrm{C}_{76} \mathrm{H}_{76} \mathrm{MgNO}_{4}$ (3•2THF) (\%): Calculated: C 83.61, H 7.02, N 1.28; Found: C 81.96; $\mathrm{H} 6.81 ; \mathrm{N} 1.57$. (Consistently low $\mathrm{C}$ analysis may be due to the formation of magnesium carbide). 
Table S1. Crystal data and refinement of 2 and 3

\begin{tabular}{|c|c|c|}
\hline & 2 & 3 \\
\hline formula & $\mathrm{C}_{78} \mathrm{H}_{74} \mathrm{IMgNO}$ & $\mathrm{C}_{68} \mathrm{H}_{64} \mathrm{MgNO}_{2}$ \\
\hline formula weight & 1192.59 & 951.51 \\
\hline crystal system & Triclinic & Monoclinic \\
\hline space group & $P-1$ & $C 2 / c$ \\
\hline$a / \AA$ & $9.3675(15)$ & $27.5368(16)$ \\
\hline$b / \AA$ & $12.9664(19)$ & $16.4904(11)$ \\
\hline $\mathrm{c} / \AA ̊$ & $26.563(4)$ & $14.7594(10)$ \\
\hline$\alpha / \operatorname{deg}$ & $90.739(7)$ & \\
\hline$\beta / \mathrm{deg}$ & $95.118(7)$ & $97.165(3)$ \\
\hline$\gamma / \operatorname{deg}$ & 101.957(8) & \\
\hline$V / \AA^{3}$ & $3142.2(8)$ & $6649.8(7)$ \\
\hline$Z$ & 2 & 4 \\
\hline$\rho_{\text {calcd }} / \mathrm{g} \cdot \mathrm{cm}^{-3}$ & 1.260 & 0.950 \\
\hline$\mu / \mathrm{mm}^{-1}$ & 3.030 & 0.064 \\
\hline$F(000)$ & 1244 & 2028 \\
\hline crystal size $/ \mathrm{mm}^{3}$ & $0.20 \times 0.10 \times 0.08$ & $0.12 \times 0.11 \times 0.08$ \\
\hline$\theta$ range/deg & $2.908-54.069$ & $2.067-27.497$ \\
\hline \multirow[t]{3}{*}{ index ranges } & $-11 \leq h \leq 11$ & $-35 \leq h \leq 35$ \\
\hline & $-14 \leq k \leq 15$ & $-20 \leq k \leq 21$ \\
\hline & $-31 \leq l \leq 31$ & $-19 \leq l \leq 15$ \\
\hline collected data & 38838 & 39691 \\
\hline unique data & $11455\left(R_{\mathrm{int}}=0.0793\right)$ & $7626\left(\mathrm{R}_{\mathrm{int}}=0.0737\right)$ \\
\hline completeness to $\theta$ & $99.7 \%$ & $99.8 \%$ \\
\hline data/restraints/parameters & $11455 / 249 / 773$ & $7626 / 271 / 376$ \\
\hline GOF on $F^{2}$ & 1.012 & 1.026 \\
\hline \multirow{2}{*}{ final $R$ indices $[I>2 \quad(I)]$} & $R_{1}=0.0658$ & $R_{1}=0.0783$ \\
\hline & $w R_{2}=0.1839$ & $w R_{2}=0.2078$ \\
\hline \multirow[t]{2}{*}{$R$ indices (all data) } & $R_{1}=0.1135$ & $R_{1}=0.1101$ \\
\hline & $w R_{2}=0.2119$ & $w R_{2}=0.2323$ \\
\hline Largest diff peak/hole $\left(\mathrm{e} \cdot \AA^{\AA^{-3}}\right)$ & $0.744 /-0.992$ & $1.155 /-0.821$ \\
\hline
\end{tabular}


EPR spectrum of 3

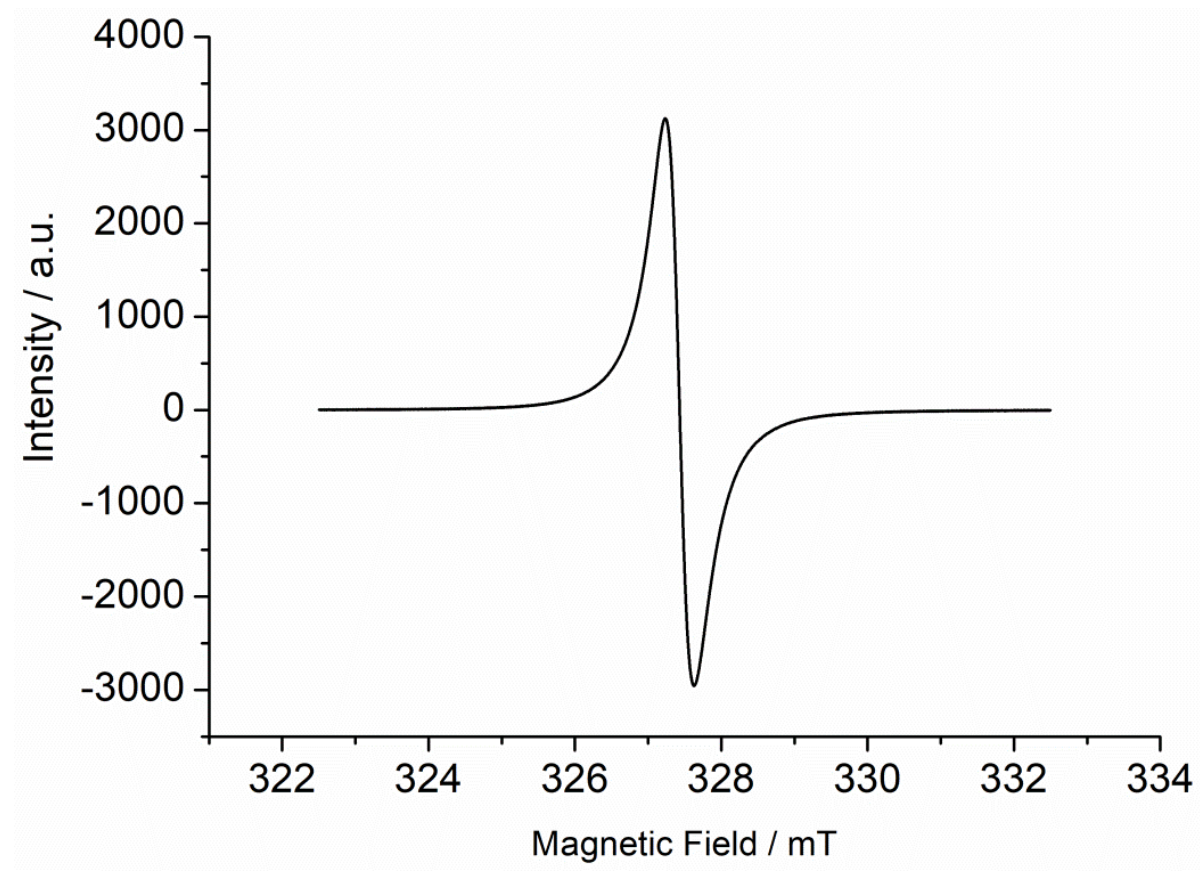

Figure S1. EPR spectrum of $\mathbf{3}$ measured in solid state at $298 \mathrm{~K}$. 
NMR spectra of 1 and 2

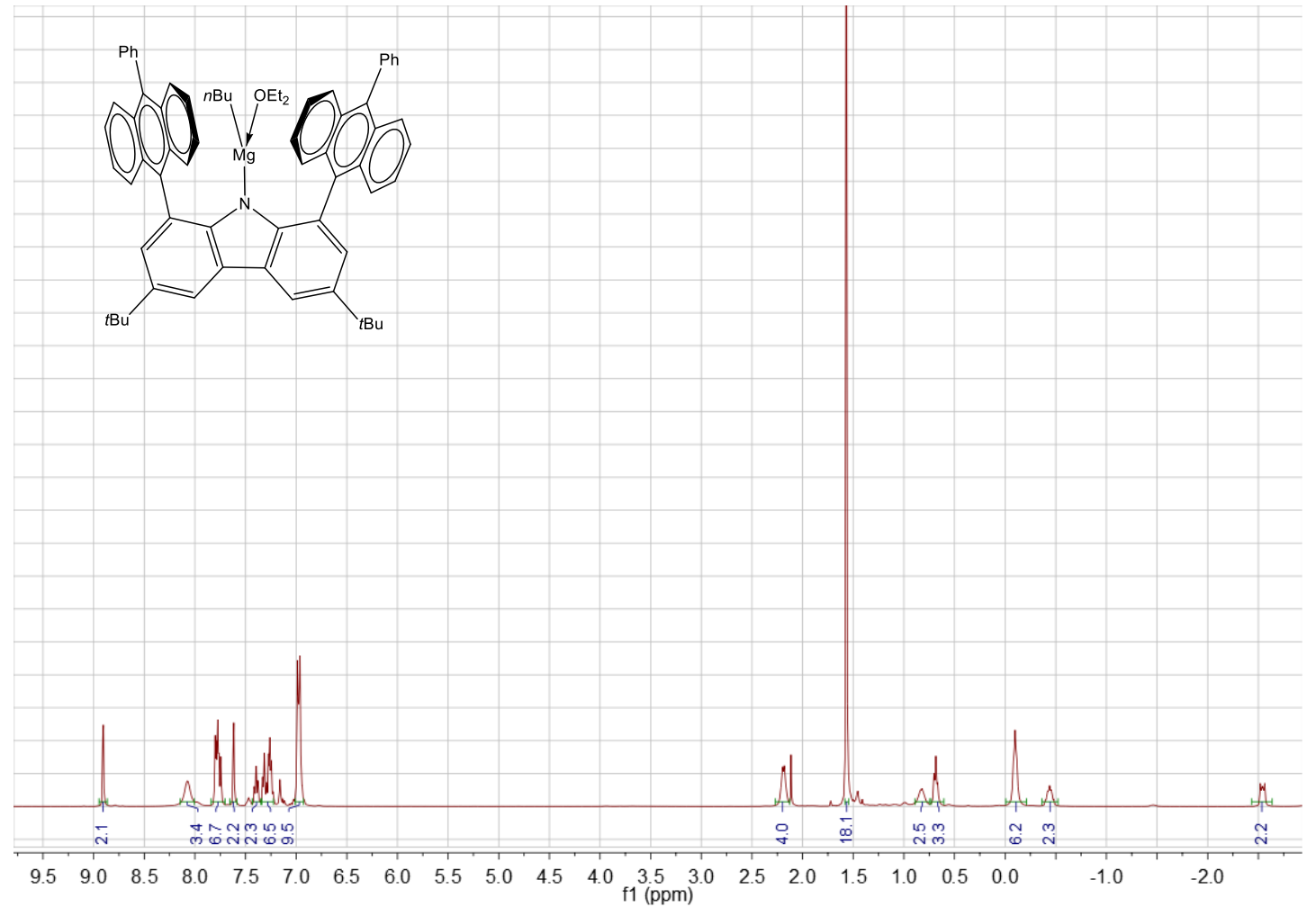

Figure S2. ${ }^{1} \mathrm{H}$ NMR spectrum of 1 in $\mathrm{C}_{6} \mathrm{D}_{6}$ at $298 \mathrm{~K}$.

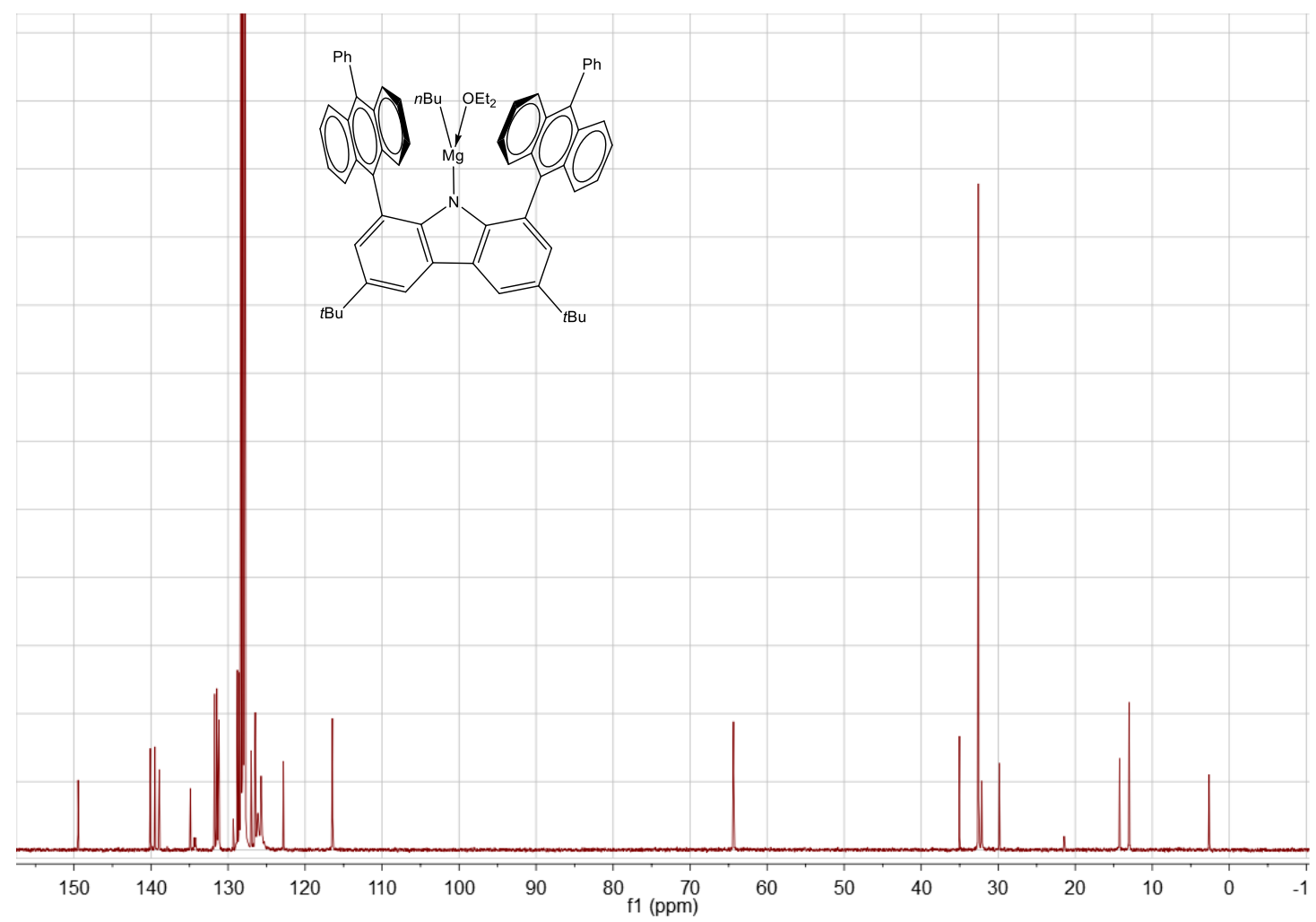

Figure S3. ${ }^{13} \mathrm{C}$ NMR spectrum of $\mathbf{1}$ in $\mathrm{C}_{6} \mathrm{D}_{6}$ at $298 \mathrm{~K}$. 


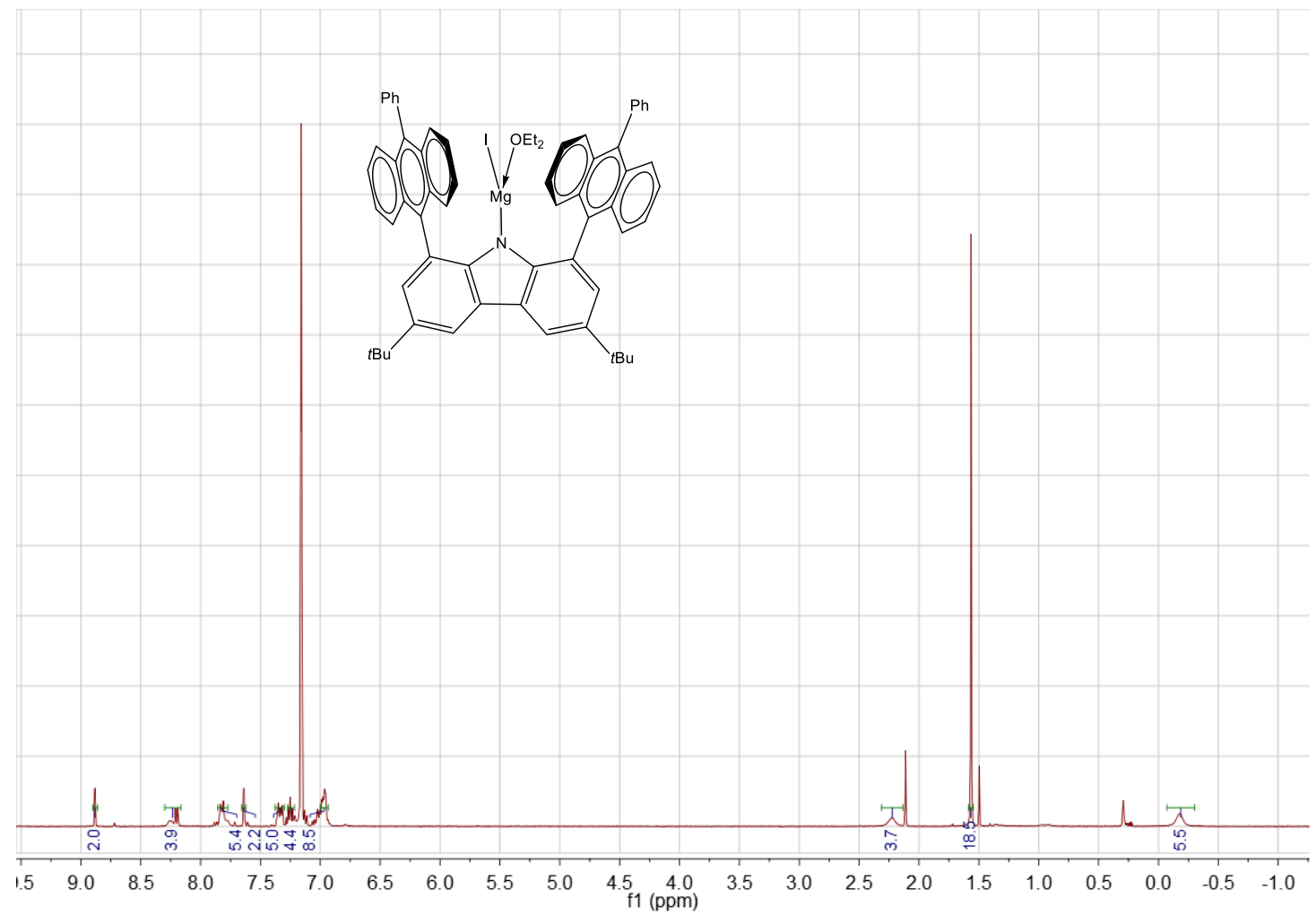

Figure S4. ${ }^{1} \mathrm{H}$ NMR spectrum of 2 in $\mathrm{C}_{6} \mathrm{D}_{6}$ at $298 \mathrm{~K}$. 


\section{Computational details}

All of the calculations were performed with the Gaussian 09 program. ${ }^{\mathrm{S} 1}$ All the geometry optimizations were performed with the hybrid B3LYP functional ${ }^{\mathrm{S} 2}$ in conjunction with a $6-311 \mathrm{G}(\mathrm{d}, \mathrm{p})^{\mathrm{S} 3}$ basis set in the gas phase. The natural bond orbital (NBO) analysis ${ }^{\mathrm{S} 4}$ was carried out at the same level. To further figure out the absorption properties of the studied complex, we also obtained the UV-Vis spectrum at the time-dependent DFT (TDDFT)//UB3LYP/6-311G(d,p) level, ${ }^{\mathrm{S} 5}$ in consideration with THF (PCM model) ${ }^{\mathrm{S} 6}$ which agrees well with the experimental data, and the calculated Kohn-Sham orbitals related to the observed transitions are shown in Table S2 and Table S3, respectively.

Table S2 Calculated absorption properties of the studied compound including wavelength (nm), oscillator strength $(f)$ and the related transition nature

\begin{tabular}{|c|c|c|c|c|}
\hline Excited-state & Wavelength/nm & $f$ & Transition nature & \\
\hline \multirow[t]{2}{*}{$\mathrm{S}_{6}$} & 567.5 & 0.06 & HOMO-1 $(\beta) \rightarrow$ LUMO $+1(\beta)$ & $65 \%$ \\
\hline & & & $\mathrm{HOMO}(\beta) \rightarrow \mathrm{LUMO}+1(\beta)$ & $30 \%$ \\
\hline $\mathrm{S}_{15}$ & 474.6 & 0.05 & $\mathrm{SOMO} \rightarrow \mathrm{LUMO}+9(\alpha)$ & $91 \%$ \\
\hline \multirow[t]{4}{*}{$\mathrm{S}_{23}$} & 406.0 & 0.20 & $\operatorname{HOMO}-3(\alpha) \rightarrow \operatorname{LUMO}(\alpha)$ & $25 \%$ \\
\hline & & & $\operatorname{HOMO}-2(\alpha) \rightarrow \operatorname{LUMO}(\alpha)$ & $24 \%$ \\
\hline & & & $\operatorname{HOMO}-3(\beta) \rightarrow \operatorname{LUMO}(\beta)$ & $28 \%$ \\
\hline & & & $\operatorname{HOMO}-2(\beta) \rightarrow \operatorname{LUMO}(\beta)$ & $21 \%$ \\
\hline
\end{tabular}


Table S3 Calculated Kohn-Sham orbitals related to the transitions

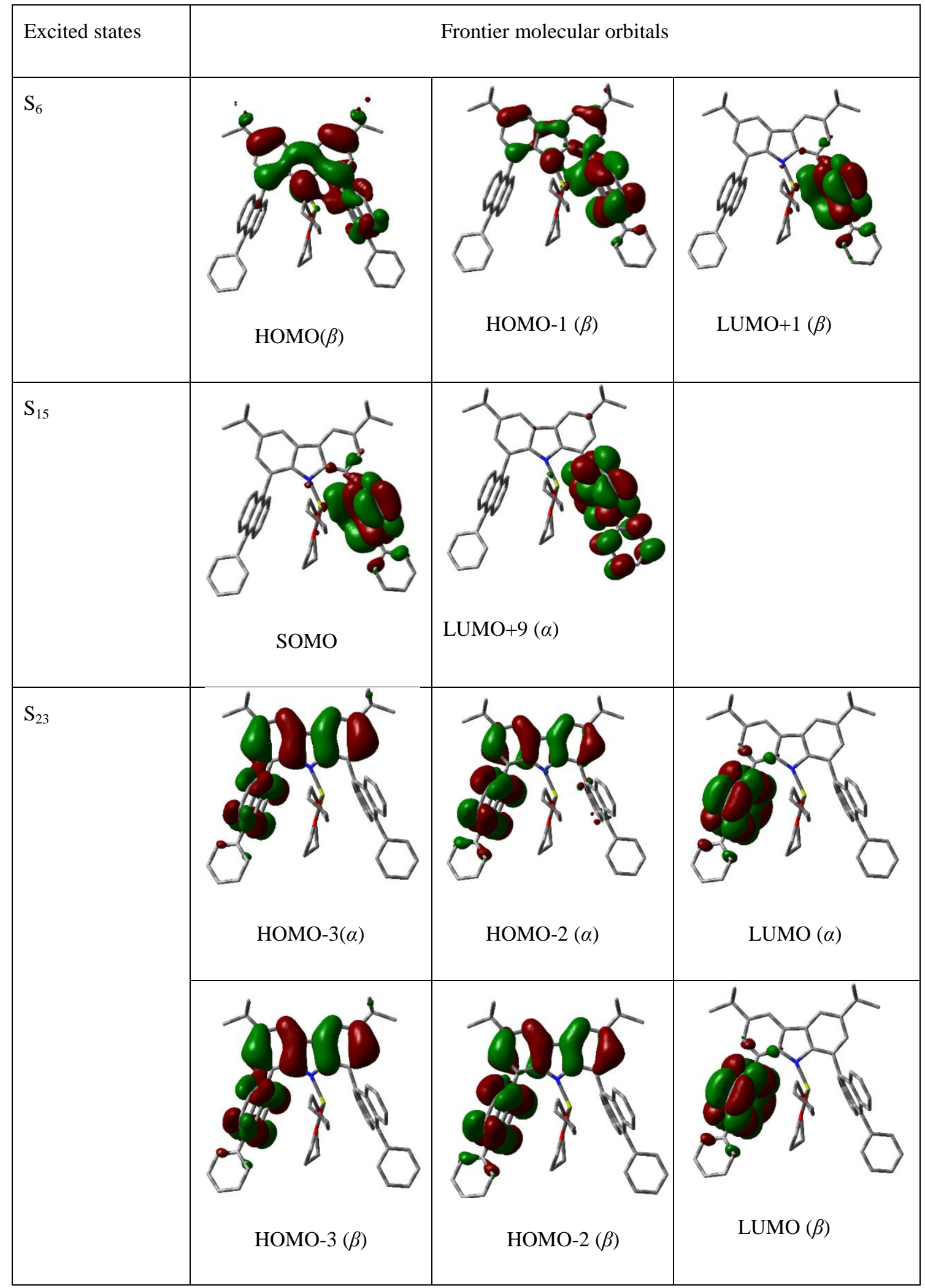




\section{References}

S1. Gaussian 09, Revision E.01, Frisch, M. J.; Trucks, G. W.; Schlegel, H. B.; Scuseria, G. E.; Robb, M. A.; Cheeseman, J. R.; Scalmani, G.; Barone, V.; Mennucci, B.; Petersson, G. A.; Nakatsuji, H.; Caricato, M.; Li, X.; Hratchian, H. P.; Izmaylov, A. F.; Bloino, J.; Zheng, G.; Sonnenberg, J. L.; Hada, M.; Ehara, M.; Toyota, M.; Fukuda, R.; Hasegawa, J.; Ishida, M.; Nakajima, T.; Honda, Y.; Kitao, O.; Nakai, H.; Vreven, T.; Montgomery, J. A., Jr.; Peralta, J. E.; Ogliaro, F.; Bearpark, M.; Heyd, J. J.; Brothers, E.; Kudin, K. N.; Staroverov, V. N.; Keith, T.; Kobayashi, R.; Normand, J.; Raghavachari, K.; Rendell, A.; Burant, J. C.; Iyengar, S. S.; Tomasi, J.; Cossi, M.; Rega, N.; Millam, J. M.; Klene, M.; Knox, J. E.; Cross, J. B.; Bakken, V.; Adamo, C.; Jaramillo, J.; Gomperts, R.; Stratmann, R. E.; Yazyev, O.; Austin, A. J.; Cammi, R.; Pomelli, C.; Ochterski, J. W.; Martin, R. L.; Morokuma, K.; Zakrzewski, V. G.; Voth, G. A.; Salvador, P.; Dannenberg, J. J.; Dapprich, S.; Daniels, A. D.; Farkas, O.; Foresman, J. B.; Ortiz, J. V.; Cioslowski, J.; Fox, D. J. Gaussian, Inc.: Wallingford CT, 2013.

S2. (a) Becke, A. D. J. Chem. Phys. 1993, 98, 5648-5652. (b) Lee, C.; Yang, W.; Parr, R. G. Phys. Rev. $\mathrm{B}, \mathbf{1 9 8 8}, 37,785-789$

S3. A McLean, D.; Chandler, G. S. J. Chem. Phys., 1980, 72, 5639-5648.

S4. Reed, A. E.; Curtiss, L. A.; Weinhold, F. Chem. Rev.,1988, 88, 899-926.

S5. Perdew, J. P.; Burke, K.; Ernzerhof, M., Phys. Rev. Lett., 1996, 77, 3865-68

S6. (a) Frediani, L.; Rinkevicius, Z.; Ågren, H., J. Chem. Phys., 2005, 122, 244104; (b) Tomasi, J.; Mennucci, B.; Cammi, R., Chem. Rev., 2005, 105, 2999-3093. 